\title{
Absolute luminescence efficiency and photonic band-gap effect of conjugated polymers with top-deposited distributed Bragg reflectors
}

\author{
Luana Persano *, Elisa Mele, Andrea Camposeo, Pompilio Del Carro, \\ Roberto Cingolani, Dario Pisignano
}

NNL, National Nanotechnology Laboratory of Istituto Nazionale di Fisica della Materia (INFM), clo Dipartimento di Ingegneria dell'Innovazione, Università di Lecce, via Arnesano, I-73100 Lecce, Italy

Received 1 March 2005; in final form 6 June 2005

Available online 5 July 2005

\begin{abstract}
We study the effects of the deposition of a photonic crystal $(\mathrm{PhC})$ structure, namely a distributed Bragg reflector, on a light-emitting conjugated polymer. The hybrid organic/ $\mathrm{PhC}$ system is realised by a novel low-temperature reactive electron beam deposition, suitable for the direct fabrication of high-reflective mirrors onto organic soft matter. We investigated the photoluminescence absolute quantum efficiency of the system, finding a yield of $30 \%$ after the mirror deposition. We found evidence for the modulation of the output spectra by the photonic band-gap of the coupled $\mathrm{PhC}$, and for the polarisation splitting (as large as $140 \mathrm{meV}$ ) of the emitted light.
\end{abstract}

(C) 2005 Elsevier B.V. All rights reserved.

\section{Introduction}

Organic conjugated materials coupled to or embedded in photonic crystals (PhCs) can provide new effective media for photonic applications. The PhCs [1] are currently of great interest due to the smart capability of controlling the emission [2], propagation [3-5], and dispersion [6] of light inside matter. These systems consist of a periodic arrangement of dielectric materials (cells), designed to show energetic band gap at the required wavelengths [1]. In particular, vertical microcavities are one-dimensional (1D) $\mathrm{PhCs}$ with one unit cell replaced by an active cell (i.e., cavity layer) of different optical thickness. Organic-based lasing microcavities centred at wavelength, $\lambda_{0}$, can be obtained by alternating $\lambda_{0} / 4$-thick layers of two different materials with sufficiently high refractive-index contrast, i.e., distributed Bragg reflectors

\footnotetext{
${ }^{*}$ Corresponding author. Fax: +390832298238.

E-mail address: luana.persano@unile.it (L. Persano).
}

(DBRs), and incorporating a single, $\lambda_{0} / 2$-thick spacer of a luminescent gain material [7,8]. The DBRs are designed in order to achieve high reflectivity $(99 \%)$ within a well-defined spectral range (stop-band), hence the lightpropagation is only allowed outside the stop-band.

The possibility of combining the optically guiding effects of PhCs with the flexibility, easy of processing and low-cost of light-emitting compounds, and particularly conjugated polymers, offers the possibility to realise novel organic-based optoelectronic devices. Unfortunately, the realisation of high-quality DBRs usually requires high deposition temperatures, which (especially if combined with oxygen atmosphere) are not compatible with organic materials, since they induce loss of adhesion to the substrate, and quenching of the optical properties [9]. Hence, organic-based lasing microcavities have been mainly fabricated by a previously realised DBR on the bottom side of the cavity and a metal mirror on the top $[10,11]$, or by sandwiching the active organics between two pre-realised dielectric mirrors [7,8]. Recently, 
we overcame this limitation by developing a low-temperature electron-beam evaporation of dielectric multistacks, thus opening the way to the realisation of monolithic organic-based microcavities comprising a top-deposited DBR. To realise fully monolithic lasers, the hybrid systems constituted by conjugated polymer and top 1D PhC deserves to be studied in depth, aiming at assessing both their fundamental properties and the influence of the evaporation process on the optical performances of the organic layer.

In this Letter, we investigate the emission properties of conjugated polymer films within a half-cavity structure made by the top-deposition of a dielectric mirror. More than the $64 \%$ of the native PL efficiency of the polymer is preserved after the evaporation process. We also evaluated the effects of the coupled PhC on the PL of the polymer, finding that the remarkable wavelength selectivity of the $\mathrm{PhC}$ greatly affects the resulting PL, enhancing the output at $1.98 \mathrm{eV}$. Finally, we found that the PL peak at lowest energy undergoes a splitting of the transversal electrical (TE) and transversal magnetic (TM) polarised light upon increasing the detection angle.

\section{Experimental}

A chloroform solution of the conjugated copolymer, poly[(9,9-dioctylfluorenyl-2,7-diyl)-co-(1,4-diphenylenevinylene-2-methoxy-5-\{2-ethylhexyloxy\}-benzene)] (PFV, Fig. 1a, American Dye Source, Canada) was spin-cast onto quartz substrates $\left(10 \times 10 \mathrm{~mm}^{2}\right)$, thus achieving films with thickness around $360 \mathrm{~nm}$. The polymer-coated substrates were then fixed on the rotating sample holder of a Temescal Supersource 2 electron-beam gun system. A base pressure of $1-2 \times 10^{-6}$ mbar was provided in the

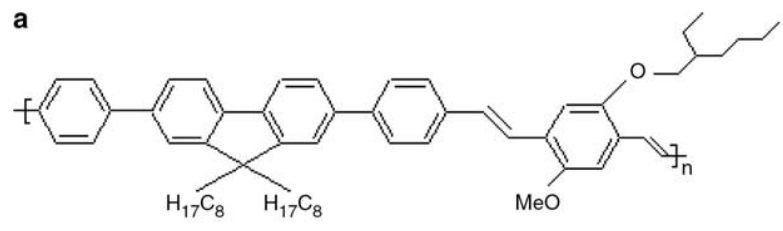

b
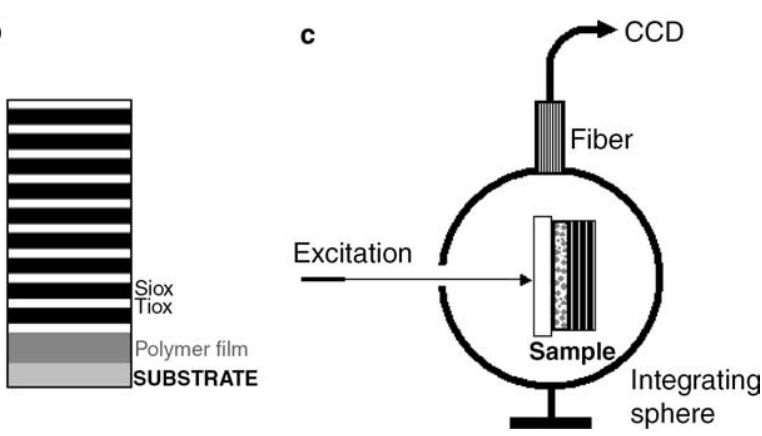

Fig. 1. (a) Molecular structure of PFV. (b) Scheme of the polymer/ PhC structure. (c) Set-up for integrating sphere measurements of the absolute quantum efficiency. chamber. The dielectric layers were deposited in an oxygen atmosphere $\left(\cong 2.4 \times 10^{-4}\right.$ mbar) using $99.9 \%$-purity $\mathrm{TiO}_{2}$ and $\mathrm{SiO}_{2}$ source materials (Leybold, Germany). The operation current was set to obtain a constant deposition rate, monitored by a quartz silver-coated crystal microbalance. $\mathrm{SiO}_{x}$ and $\mathrm{TiO}_{x}$ films were deposited at low temperature, the only chamber heating being induced by the electronic gun. The chamber was at room temperature (about $20^{\circ} \mathrm{C}$ ) at the beginning of the deposition, reaching less than $70{ }^{\circ} \mathrm{C}$ at the end of the process. The dielectric mirror was composed of 9.5 pairs of $\mathrm{TiO}_{x} / \mathrm{SiO}_{x}$ layers. The achieved thickness and refractive index at $\lambda=550 \mathrm{~nm}$ were of $69 \mathrm{~nm}$ and 1.99 , and $96 \mathrm{~nm}$ and 1.42 , for the titania and the silica layers, respectively. The resulting conjugated polymer/PhC architecture is schematised in Fig. 1b. In the same evaporation run, a reference DBR was deposited on a quartz substrate.

The stop-bands of our mirrors were determined by transmission measurements by a Cary 5000 spectrophotometer (Varian, Australia). The PL and the absolute quantum efficiency, $\eta_{\mathrm{PL}}$, of the polymer film were measured both before and after the evaporation process by a fibre-coupled charge coupled device (CCD, Ocean Optics, FL), by exciting with a $\mathrm{He}-\mathrm{Cd}$ laser $(\lambda=$ $325 \mathrm{~nm}) . \eta_{\mathrm{PL}}$ was determined by placing the sample in an integrating sphere.

\section{Results and discussion}

So far, the high temperature needed for realising dielectric reflectors has strongly limited the possibility of implementing monolithic, integrated inorganic/organic devices, such as vertical surface-emitting lasers, solar cells and hybrid PhCs. In particular, the oxygen atmosphere needed for reactive growth of dielectric oxides is known to quench the luminescence of organic molecules, particularly through the carbonyl $(\mathrm{C}=\mathrm{O})$ substitution of the vinyl group in poly(phenylenevinylene) compounds [8]. Indeed, such a quenching of the luminescence, $I$, has been effectively explained in terms of exciton diffusion towards oxygen-induced defects, and described by a simple diffusion model:

$I(t) \cong I_{0} t^{1 / 2} \exp \left(-\frac{t}{\tau_{\mathrm{d}}}\right)^{1 / 3} \exp \left(-\frac{t}{\tau_{0}}\right)$

where $t$ is the time, $\tau_{\mathrm{d}}$ and $\tau_{0}$ are the exciton diffusion and life characteristic times, respectively, and $I_{0}$ is a constant [9]. In addition, temperatures higher than the glass-transition temperature of the organic compound would cause an irreversible loss of adhesion of the polymer from its substrate. For most of conjugated polymers, the glass-transition, and the degradation of the emission properties, occur between 100 and $250{ }^{\circ} \mathrm{C}$ $[9,12]$. To overcome this difficulty, we developed a 
low-temperature $\left(T<70{ }^{\circ} \mathrm{C}\right)$ electron-beam deposition method for the direct realisation of 1D PhCs onto conjugated films.

\subsection{Determination of absolute quantum PL efficiency}

In a standard measurement on bare conjugated polymers [13], $\eta_{\mathrm{PL}}$ is determined by exciting the samples with the laser incident on the organic layer. One accounts for those photons which are not absorbed by the sample at their first incidence, and are absorbed after the successive reflections on the surface of the integrating sphere

$\eta_{\mathrm{PL}}=\frac{P_{1}-P_{\mathrm{D}}}{(1-R-T)(1-\alpha) X_{\mathrm{L}}}$,

where $R$ and $T$ indicate the reflectance and the transmittance of the film, respectively, and $\alpha$ is the substrate absorption. $X_{\mathrm{L}}$ stands for the excitation signal, measured with the laser incident on the sphere and without sample, $P_{1}$ is the PL signal measured with the laser beam incident on the sample (measured by directing the laser on the film) while $P_{\mathrm{D}}$ is the PL accounting for photons emitted after the first incidence, i.e.,

$P_{\mathrm{D}}=(1-\alpha)^{2}(R+T) P_{2}$,

where $P_{2}$ is the PL signal with the laser beam incident on the sphere wall. After placing the sample in the sphere, we measured $P_{2}$, by directing the excitation on the sphere wall, and $P_{1}$, by directing the laser on the film. Finally, we removed the sample and measured $X_{\mathrm{L}}$. All the signals were normalised by the spectral response of the experimental set-up.

In order to assess the influence of the deposition process on the luminescence efficiency of the active material, we measured $\eta_{\mathrm{PL}}$ before, and after, the growth of the top dielectric mirror. While the measurement of $\eta_{\mathrm{PL}}$ on conjugated materials is a well-established technique [13], a specific procedure has been implemented for determining the absolute quantum yield of the organics/PhC system (excitation geometry in Fig. 1c). In fact, the top dielectric mirror changes the path of excitation photons which are not absorbed by the sample at their first incidence; hence one has to consider a different expression for $P_{\mathrm{D}}$, instead of that of Eq. (2). By neglecting the absorption of light from the DBR $\left(R_{\mathrm{DBR}}+T_{\mathrm{DBR}}=1\right)$, the excitation intensity absorbed by the sample after diffusion with the laser beam on the sample $\left(X_{\mathrm{ABS}}\right)$, can be related to the intensity absorbed by the sample after diffusion with the laser beam incident on the sphere, $\left(X_{\mathrm{ABS}, \mathrm{Sph}}\right)$ : $X_{\mathrm{ABS}}=\left[(1-\alpha)^{2}\left(R+R_{\mathrm{DBR}} T^{2}\right)+(1-\alpha) T T_{\mathrm{DBR}}\right] X_{\mathrm{ABS}, \mathrm{Sph}}$. The sum, $\left[(1-\alpha)^{2}\left(R+R_{\mathrm{DBR}} T^{2}\right)+(1-\alpha) T T_{\mathrm{DBR}}\right]$ (hereafter labelled as $A+B)$, represents the amount of photons not absorbed by the sample at their first incidence. Hence, $P_{\mathrm{D}}$ can be expressed as

$P_{\mathrm{D}}=(A+B) P_{2}$ and the $\eta_{\mathrm{PL}}$ for the organics with the top-deposited $\mathrm{PhC}$ becomes

$\eta_{\mathrm{PL}}=\frac{P_{1}-(A+B) P_{2}}{(1-R-T)(1-\alpha) X_{\mathrm{L}}}$.

The measured $\eta_{\mathrm{PL}}$ for the native conjugated polymer film is $47 \%$. The same sample, after the direct electron-beam deposition of the dielectric mirror (stop-band of about $145 \mathrm{~nm}$ and maximum reflectivity larger than $99.5 \%$, Fig. 2a), exhibit a value of the PL efficiency of about $30 \%$. We attribute the observed decrease of $\eta_{\mathrm{PL}}$ to the mild heating induced by the electron-gun during the evaporation, however we note that the resulting efficiency is still considerably high for conjugated polymers in the solid-state [13]. The same evaporated dielectric stack likely acts as protecting encapsulation layer, preventing a further decrease of the luminescence yield during the exposure at the oxygen atmosphere of the deposition chamber. The final value of $\eta_{\mathrm{PL}}$ is fully compatible with the realisation of organic-based photonic devices.

\subsection{Spectral properties}

In order to have a deeper insight into the spectral properties of our system, we investigated the PL spectra and their angular dependence. The native polymer film exhibits a single broad PL feature peaked at $2.40 \mathrm{eV}$,

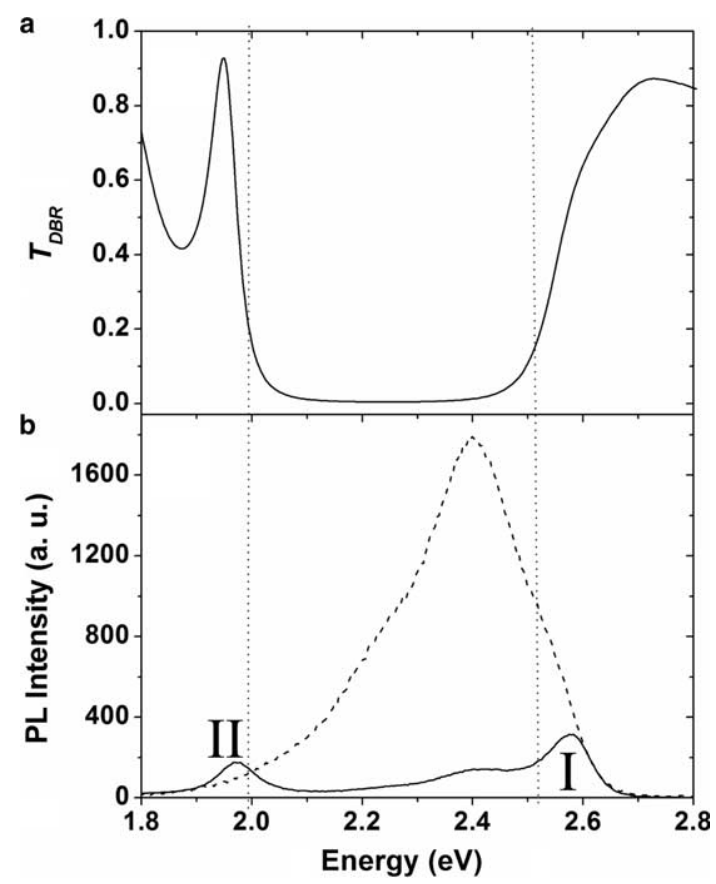

Fig. 2. (a) Transmission $\left(T_{\mathrm{DBR}}\right)$ spectrum of the DBR evaporated on top of the polymer film. (b) PL spectra of the native polymer film (dotted line) and of the organic/PhC system (continuous line) $\left(\Theta=0^{\circ}\right.$ ), normalised to the same acquisition time. I and II indicate the highenergy and the low-energy peak of the PL from the polymer/PhC, respectively, collected after passing through the DBR. It can be appreciated that only the peak II shows an enhancement with respect to the bare conjugated polymer. 
with a full-width at half maximum (FWHM) of about $300 \mathrm{meV}$ (Fig. 2b). Upon the deposition of the top mirror, the overall emission is dramatically changed, being modulated by the PhC band-gap (Fig. 2b). Indeed, the transmitted PL is split into two main branches by the stop-band, and three resulting peaks can be appreciated (Fig. 2b). In the region of forbidden light-propagation $(2.0-2.5 \mathrm{eV})$, the main emission peak of the polymer is strongly attenuated (the intensity reduction at $2.40 \mathrm{eV}$ is about $92 \%$ with respect to the bare conjugated film). Two main peaks arise instead at the low- and at the high-energy tails of the photonic band-gap, that filters the emission of the polymer, thus determining a remarkable line-narrowing of the resulting emission. The highenergy PL maximum (peak I in Fig. 2b) is at about $2.58 \mathrm{eV}$ (FWHM $\cong 100 \mathrm{meV}$ ), whereas, the low-energy peak (peak II in Fig. 2b, FWHM $\cong 80 \mathrm{meV}$ ) is at $1.98 \mathrm{eV}$ (where the PhC reflectivity decreases to $57 \%$ ). The low-energy peak of the half-cavity is enhanced with respect to the corresponding emission of the native film by a factor around 1.7.

The fact that the PL enhancement only takes place for the low-energy peak would rule out the reflection of part of the excitation photons back into the active polymer as reason of the observed enhancement. In fact, such mechanism should enhance both the peak I and the peak II with respect to the native PL. This conclusion is also supported by the PL of the polymer/mirror system, collected by employing three different excitation wavelengths (430, 405 and $395 \mathrm{~nm}$ ), which do not exhibit significant differences (Fig. 3a). This finding confirms that the enhancement at $1.98 \mathrm{eV}$ is not related to the reflection back into the polymer of the excitation photons, since different pump wavelengths (corresponding to different values of the mirror reflectivity) would otherwise determine different intensities of the overall emission spectra.

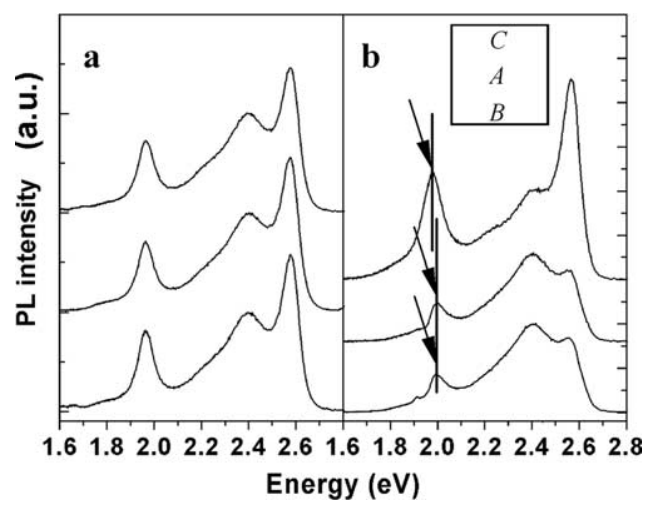

Fig. 3. (a) PL spectra of the organic/PhC system $\left(\Theta=0^{\circ}\right)$, collected by exciting at different wavelength (from bottom to top $\lambda=430,405$ and $395 \mathrm{~nm}$ ). All the spectra have been collected with the same acquisition time. (b) PL spectra of the organic/PhC system $\left(\Theta=0^{\circ}\right)$, collected by exciting three sample points (inset): (from top to bottom A, C and B) with different thickness of the active layer. The vertical lines and the arrows indicate the position of the peak II.
We believe that the enhancement at $1.98 \mathrm{eV}$ is attributable to the coupling of the spontaneous emission of the conjugated polymer to the resonances of the cavity-like structure air/quartz/polymer/PhC [14]. Indeed, the achieved thickness of the active polymer $(d \cong 360$ $\mathrm{nm})$ well-matches the resonance condition for $1.98 \mathrm{eV}$, i.e., $2 n d=m \lambda$, with $m=2$. To get a deeper insight into this issue, we collected the PL from the organics/PhC system by exciting different points of the sample, with slightly different values of the polymer thickness (this is in fact due to the non-perfectly uniform thickness achievable by spin-coating). We found that, upon varying the thickness of the active layer, the peak I does not exhibit shift in wavelength (Fig. 3b). On the contrary, the energy of the peak II shifts of about $20 \mathrm{meV}$ (Fig. $3 \mathrm{~b}$ ), corresponding to a change of the polymer thickness of about $4 \mathrm{~nm}$. Such shift due to the change of the thickness confirms the effect of resonance within the air/ quartz/polymer/PhC architecture.

Finally, we studied the angular dependence of the emitted light. We found that the peak I rapidly blueshifts outside from the high-energy tail of the spontaneous emission spectrum upon increasing $\Theta$, whereas the peak II splits into a doublet (IIa and IIb) for $\Theta>40^{\circ}$ (Fig. 4). After splitting, the component IIa is TE-polarised, whereas the IIb is TM-polarised (inset of Fig. 4). In one-dimensional microcavities, this effect is attributed to the marked dependence of the light penetration inside the mirror on the polarisation [15]. The here observed energy difference between the TE and TM peaks is about $51 \mathrm{meV}$ at $\Theta=45^{\circ}$, grows almost linearly up to $140 \mathrm{meV}$ at $\Theta=65^{\circ}$, and then saturates. Due the relevant interest of microcavities based on conjugated polymers for highefficient light emitting devices, photodiodes, and vertical cavity surface-emitting lasers, the development of novel architectures exhibiting polarisation splitting could have great importance for the realisation of polarised organic-based optoelectronics.

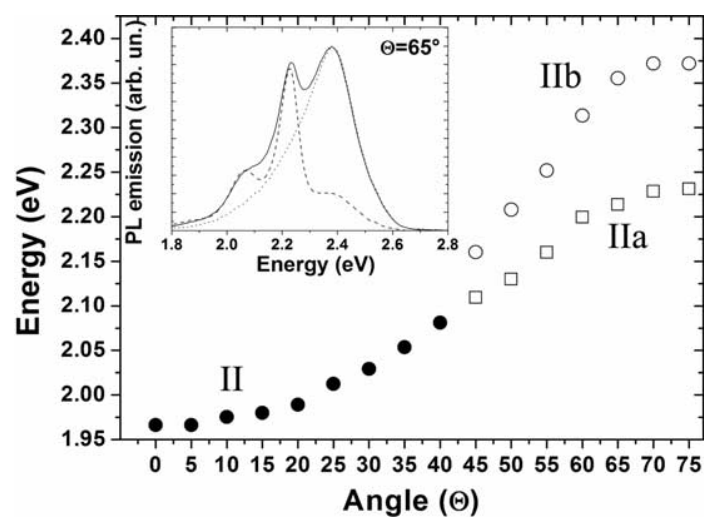

Fig. 4. Angular dependence of peak II. Full circles: main peak $\left(0^{\circ} \leqslant \Theta \leqslant 40^{\circ}\right)$, squares: TE peak, empty circles: TM peak $\left(40^{\circ} \leqslant \Theta \leqslant 75^{\circ}\right)$. Inset: PL spectra with unpolarised light (continuous line), TE (dashed line), and TM (dotted line) polarisation. $\left(\Theta=65^{\circ}\right)$. 


\section{Conclusion}

We investigated the absolute quantum efficiency and the emission properties of a hybrid architecture made by a conjugated polymer and a top-deposited $\mathrm{PhC}$. We found a value of $\eta_{\mathrm{PL}}$ as high as $30 \%$ after the mirror deposition, which is fully compatible with the realisation of photonic devices. The changes of the PL induced by the $\mathrm{PhC}$, and the PL dependence on the detection angle, were determined. We found evidence for remarkable wavelength selectivity, induced on the spontaneous emission of the conjugated compound by the coupled $\mathrm{PhC}$, that dramatically changes the resulting PL, enhancing the output at $1.98 \mathrm{eV}$. Finally, we observed a polarisation-induced energy splitting of the TE and TM peaks, as large as $140 \mathrm{meV}$. These findings suggest a close resemblance of the air/quartz/polymer/PhC architecture with 1D microcavities.

\section{References}

[1] J.D. Joannopoulos, R.D. Meade, J.N. Winn, Photonic Crystals: Molding the Flow of Light, Princeton University Press, Cambridge, MA, 1995.

[2] E. Yablonovitch, Phys. Rev. Lett 58 (1987) 2059.
[3] A. Mekis, J.C. Chen, I. Kurland, S. Fan, P.R. Villeneuve, J.D. Joannopoulos, Phys. Rev. Lett. 77 (1996) 3787.

[4] S.G. Johnson, S. Fan, P.R. Villeneuve, J.D. Joannopoulos, L.A. Kolodziejski, Phys. Rev. B 60 (1999) 5751.

[5] S.G. Johnson, P.R. Villeneuve, S. Fan, J.D. Joannopoulos, Phys. Rev. B 62 (2000) 8212.

[6] B. Gralak, S. Enoch, G. Tayeb, J. Opt. Soc. Am. A 17 (2000) 1012.

[7] T. Granlund, M. Theander, M. Berggren, M. Andersson, A. Ruzeckas, V. Sundström, G. Björk, M. Granström, O. Inganäs, Chem. Phys. Lett. 288 (1998) 879.

[8] M. Theander, T. Granlund, D.M. Johanson, A. Ruseckas, V. Sundström, M.R. Andersson, O. Inganäs, Adv. Mater. 13 (2001) 323.

[9] F. Papadimitrakopoulos, K. Konstadinidis, T.M. Miller, R. Opila, E.A. Chandross, M.E. Galvin, Chem. Mater. 6 (1994) 1563.

[10] V. Bulović, V.G. Kozolov, V.B. Khalfin, S.R. Forrest, Science 279 (1998) 553.

[11] N. Tessler, G.J. Denton, R.H. Friend, Nature 382 (1996) 695.

[12] D. Pisignano, L. Persano, R. Cingolani, G. Gigli, F. Babudri, G.M. Farinola, F. Naso, Appl. Phys. Lett. 84 (2004) 1365.

[13] N.C. Greenham, I.D.W. Samuel, G.R. Hayes, R.T. Philips, Y.A.R.R. Kessener, S.C. Moratti, R.H. Friend, Chem. Phys. Lett. 241 (1995) 89.

[14] F. Hide, M.A. Díaz-García, B.J. Schwartz, M.R. Andersson, Q. Pei, A.J. Heeger, Science 273 (1996) 1833.

[15] G. Panzarini, L.C. Andreani, A. Armitage, D. Baxter, M.S. Skolnick, V.N. Astratov, J.S. Roberts, A.V. Kavokin, M.R. Vladimrova, M.A. Kaliteevsky, Phys. Rev. B 59 (1999) 5082. 